PROCEEDINGS OF THE

AMERICAN MATHEMATICAL SOCIETY

Volume 132, Number 12, Pages 3689-3695

S 0002-9939(04)07488-X

Article electronically published on July 20, 2004

\title{
THE COMPLEXITY OF THE WORD-PROBLEM FOR FINITE MATRIX RINGS
}

\author{
CSABA SZABÓ AND VERA VÉRTESI
}

(Communicated by Lance W. Small)

\begin{abstract}
We analyze the so-called word-problem for $M_{2}\left(Z_{2}\right)$, the ring of $2 \times 2$ matrices over $Z_{2}$. We prove that the term-equivalence problem for the semigroup (and so for the ring) $M_{2}\left(Z_{2}\right)$ is coNP-complete.
\end{abstract}

\section{INTRODUCTION}

In this paper we study the computational complexity of the word-problem for $M_{2}\left(Z_{2}\right)$. We shall use the standard notation for computational complexity, as $\mathrm{P}$, $\mathrm{NP}$, coNP, etc.

The word-problem for an algebra $\mathcal{A}$ has two different versions for terms and for polynomials. We call an expression term if it contains only variables and we call it polynomial if it may contain elements of $\mathcal{A}$. The term-equivalence problem over $\mathcal{A}$ (TERM-EQ $\mathcal{A}$ ) asks whether two given terms agree for every substitution. For example $x^{6}$ and $i d$ are terms over the group $S_{3}$, and they are equivalent because the exponent of $S_{3}$ is 6 . The polynomial-equivalence problem (POL-EQ $\mathcal{A}$ ) asks the same for polynomials, for example $x(1,2) y x^{2}(1,2,3)$ and $x^{2} y$ are polynomials over the group $S_{3}$, but they are not equivalent, e.g. by substituting $x=y=i d$ the two values are not equal. Here $(1,2)$ denotes the transposition flipping 1 and 2 and $(1,2,3)$ denotes the 3 -cycle mapping 1 to 2,2 to 3 and 3 to 1 .

Let TERM-SAT $\mathcal{A}$ and POL-SAT $\mathcal{A}$ denote the term- and polynomial-satisfiability problems, respectively. The instance of TERM-SAT (POL-SAT) is a term (polynomial) $t$ and an element $a \in \mathcal{A}$. The question is whether there is an evaluation of $t$ such that $t=a$. Observe that for any finite algebra, TERM-EQ and POL-EQ are both in coNP and TERM-SAT (POL-SAT) is in NP.

\section{Preliminaries}

We present a few recent results for some algebraic structures.

It is already known [3] that for a commutative ring $\mathcal{R}$ the TERM-EQ problem is in $\mathrm{P}$ if $\mathcal{R}$ is nilpotent and coNP-complete otherwise. Burris and Lawrence proved in 2 that the same holds for rings in general. Following their proof it is easy to see that for a nilpotent ring $\mathcal{R}$ the problem POL-EQ $\mathcal{R}$ is in P. A straightforward

Received by the editors September 5, 2002 and, in revised form, July 24, 2003.

2000 Mathematics Subject Classification. Primary 68Q17, 03C13.

Key words and phrases. 0-simple semigroup, term, complexity.

(C)2004 American Mathematical Society 
consequence of their result is that if the ring is not nilpotent, then POL-EQ $\mathcal{R}$ is coNP-complete.

So, for example, for the ring $Z_{2}$, the coNP-completeness of TERM-EQ $Z_{2}$ is an easy consequence of the NP-completeness of 3-SAT. But the proof uses high powers of sums. This is the reason why Willard and Lawrence introduced the $\Sigma$ version of the problems, where every polynomial is a sum of monomials, e.g. an expression of the form $(x+y)^{n}$ is too long when expanded. The TERM ${ }_{\Sigma}$-EQ $\left(\mathrm{POL}_{\Sigma}-\mathrm{EQ}\right)$ problem asks whether two terms (polynomials), $p$ and $q$ - that are sums of monomials are equal at every substitution. They proved in [5] that

Theorem 1. Let $\mathcal{R}$ be a ring and $\mathbf{J}(\mathcal{R})$ denote its Jacobson radical.

If $\mathcal{R} / \mathbf{J}(\mathcal{R})$ is commutative, then $\mathrm{TERM}_{\Sigma}-\mathrm{EQ} \mathcal{R}$ is in $P$.

If $\mathcal{R}=M_{n}(F)$ is a finite matrix ring whose invertible elements form a nonsolvable group, then $\mathrm{TERM}_{\Sigma}-\mathrm{EQ} \mathcal{R}$ is coNP-complete. That is, if $n \geq 3$ or $|F| \geq 4$, then $\mathrm{TERM}_{\Sigma}-\mathrm{EQ} M_{n}(F)$ is coNP-complete.

They ask what happens for $n=2$ and $|F|=2,3$ (see Problem 2 in [5]). We examine this question in Section 4 .

The group case is only partially solved. An unpublished result of Lawrence and Burris is the following:

Theorem 2. Let $\mathcal{G}$ be a group. If $\mathcal{G}$ is nilpotent, then TERM-EQ $\mathcal{G}$ is in P. If $\mathcal{G}$ is non-solvable, then TERM-EQ $\mathcal{G}$ is coNP-complete.

The answer for semigroups is less complete. In [1] the authors show for a special class of aperiodic monoids that the POL-EQ problem is tractable. In [7] the authors prove that

Theorem 3. POL-EQ $M_{n}(F)$ and POL-SAT $M_{n}(F)$ are coNP-complete.

It is also shown that

Theorem 4. Let $S$ be a combinatorial 0-simple semigroup. Then POL-EQ $S$, POL-SAT $S$, TERM-EQ $S$, and TERM-SAT $S$ are in $P$.

In [6] V. Yu. Popov and M. V. Volkov exhibit a semigroup of size $\leq 2^{1700}$ with a coNP-complete TERM-EQ problem. Later Kisieliewicz in [4] presented an example of size a few hundred. In this paper we investigate the word-problem for the matrix semigroup $M_{2}\left(Z_{2}\right)$. We prove in Section 4 that

Theorem 5. TERM-EQ is coNP-complete for the semigroup $M_{2}\left(Z_{2}\right)$.

This result not only provides a 16 element example of a semigroup with coNPcomplete word problem that is significantly smaller than the previously known examples, but also, as an easy corollary we get that the TERM ${ }_{\Sigma}$-EQ is coNPcomplete for the matrix ring as well. Moreover, following the idea of the proof we exhibit an example of a semigroup of size 13 with a coNP-complete word problem.

\section{Combinatorial COMPletely 0-Simple SEmigroups}

We give a description of combinatorial completely 0 -simple semigroups. Let $M$ be a 0-1 matrix such that each row and column contains at least one 1 entry. We define $S=S_{M}$, the completely 0 -simple semigroup belonging to the regular matrix $M$. Let $\Lambda$ and $I$ denote the index set for the rows and columns of $M$. The 
underlying set of $S$ consists of all pairs of the form $\langle i, \lambda\rangle$ where $i \in I$, and $\lambda \in \Lambda$, along with 0 . An associative multiplication is given by the following rule:

$$
\langle i, \lambda\rangle\langle j, \mu\rangle= \begin{cases}\langle i, \mu\rangle & \text { if } M(\lambda, j)=1, \\ 0 & \text { otherwise. }\end{cases}
$$

Note, that for $a \in S_{M}, a^{2}=a$ or 0 according to the matrix $M$, and for every case $a^{2}=a^{3}=a^{4}=\ldots$

Example 6. Let

$$
A=\left(\begin{array}{lll}
0 & 1 & 1 \\
1 & 0 & 1 \\
1 & 1 & 0
\end{array}\right)
$$

where $\Lambda=I=\{1,2,3\}$. Then $\langle 1,2\rangle\langle 3,1\rangle=\langle 1,1\rangle$ as $A(2,3)=1$ and $\langle 1,2\rangle\langle 2,1\rangle=0$ as $A(2,2)=0$. In $S_{A}$ the product $\langle i, \lambda\rangle\langle j, \mu\rangle=0$ if and only if $\lambda=j$.

Note that the completely 0-simple semigroups are also called Rees-matrix semigroups. We continue with an observation:

Lemma 7. Let $S=S_{M}$ be a combinatorial Rees-matrix semigroup with elements $a_{1}, \ldots, a_{n} \in S$, where $a_{j}=\left\langle i_{j}, \lambda_{j}\right\rangle$ for $1 \leq j \leq n$.

(1) $a_{1} \cdots a_{n}=0$ if and only if there exists $a k, 1<k \leq n$, such that $a_{k-1} a_{k}=0$.

(2) If $a_{1} \cdots a_{n} \neq 0$, then $a_{1} \cdots a_{n}=\left\langle i_{1}, \lambda_{1}\right\rangle \cdots\left\langle i_{n}, \lambda_{n}\right\rangle=\left\langle i_{1}, \lambda_{n}\right\rangle$.

\section{The Semigroup $M_{2}\left(Z_{2}\right)$}

We split the semigroup $M_{2}\left(Z_{2}\right)$ into two parts: the group of invertible matrices and the multiplicative semigroup of the 10 singular matrices.

The group of invertible matrices is isomorphic to the symmetric group $S_{3}$. An isomorphism is given by simply the action of the matrix on the 3 nonzero vectors of the vectorspace $Z_{2}^{2}:\left(\begin{array}{l}0 \\ 1\end{array}\right),\left(\begin{array}{l}1 \\ 0\end{array}\right),\left(\begin{array}{l}1 \\ 1\end{array}\right)$ :

$$
\begin{aligned}
\left(\begin{array}{ll}
1 & 0 \\
0 & 1
\end{array}\right) \rightarrow i d & \left(\begin{array}{ll}
1 & 1 \\
0 & 1
\end{array}\right) \rightarrow(1,3) & \left(\begin{array}{ll}
1 & 0 \\
1 & 1
\end{array}\right) \rightarrow(2,3) \\
\left(\begin{array}{ll}
1 & 1 \\
1 & 0
\end{array}\right) \rightarrow(1,2,3) & \left(\begin{array}{ll}
0 & 1 \\
1 & 1
\end{array}\right) \rightarrow(1,3,2) & \left(\begin{array}{ll}
0 & 1 \\
1 & 0
\end{array}\right) \rightarrow(1,2) .
\end{aligned}
$$

The semigroup of singular matrices $(L)$ is isomorphic to the combinatorial 0simple semigroup $S_{A}$, where $A$ was defined in Example [6 Indeed, let $v_{1}=u_{2}=$ $\left(\begin{array}{l}1 \\ 0\end{array}\right), v_{2}=u_{1}=\left(\begin{array}{l}0 \\ 1\end{array}\right), v_{3}=u_{3}=\left(\begin{array}{l}1 \\ 1\end{array}\right) \in Z_{2}^{2}$.

Let us define the map $\phi$ from the semigroup of singular matrices in $M_{2}\left(Z_{2}\right)$ to $S_{A}$ in the following way:

$$
\phi\left(v_{i} \cdot u_{\lambda}^{T}\right)=\langle i, \lambda\rangle \text { and } \phi\left(\left(\begin{array}{ll}
0 & 0 \\
0 & 0
\end{array}\right)\right)=0 .
$$

Here, $u_{\lambda}^{T} \cdot v_{j}=\left\{\begin{array}{ll}0 & \text { if } \lambda=j \\ 1 & \text { if } \lambda \neq j .\end{array}\right.$ Hence

$$
\left(v_{i} \cdot u_{\lambda}^{T}\right) \cdot\left(v_{j} \cdot u_{\mu}^{T}\right)=v_{i} \cdot\left(u_{\lambda}^{T} \cdot v_{j}\right) \cdot u_{\mu}^{T}= \begin{cases}v_{i} \cdot u_{\mu}^{T} & \text { if } u_{\lambda}^{T} \cdot v_{j}=1 \\ 0 & \text { if } u_{\lambda}^{T} \cdot v_{j}=0 .\end{cases}
$$


This verifies that $\phi$ is an isomorphism between the multiplicative semigroup of singular matrices of $M_{2}\left(Z_{2}\right)$ and $S_{A}$. These two isomorphisms extend a map between $M_{2}\left(Z_{2}\right)$ and $S_{3} \cup S_{A}$ that is an isomorphism where the multiplication between the elements of $S_{3}$ and $S_{A}$ is defined as follows. For $\pi \in S_{3}$ and $\langle i, j\rangle \in S_{A}$ let

$$
\langle i, \lambda\rangle \pi=\langle i, \pi(\lambda)\rangle \quad \text { and } \quad \pi\langle i, \lambda\rangle=\left\langle\pi^{-1}(i), \lambda\right\rangle
$$

It is easy to see that

Lemma 8. Let $m_{1}, \ldots, m_{n} \in M_{2}\left(Z_{2}\right)=S_{3} \cup S_{A},\langle i, \lambda\rangle,\langle j, \mu\rangle \in S_{A}$ and $\pi \in S_{3}$.

(1) $m_{1} \cdots m_{n} \in S_{A}$ if and only if there exists a $k$ for which $m_{k} \in S_{A}$, i.e. a product is in $S_{A}$ if and only if at least one of the factors is in $S_{A}$.

(2) $\langle i, \lambda\rangle \pi\langle j, \mu\rangle=0$ if and only if $\pi(\lambda)=j$.

We shall need the analogue of Lemma 7.

Lemma 9. Let $a_{1}, \ldots, a_{n} \in S_{A}$ where $a_{j}=\left\langle i_{j}, \lambda_{j}\right\rangle$ for $1 \leq j \leq n$ and $\pi_{1}, \pi_{2}, \ldots$, $\pi_{n+1} \in S_{3}$. Then

(1) $\pi_{1} a_{1} \pi_{2} a_{2} \cdots \pi_{n} a_{n} \pi_{n+1}=0$ if and only if there exists $k, 1<k \leq n$, such that $a_{k-1} \pi_{k} a_{k}=0$ (i.e. $\pi_{k}\left(\lambda_{k-1}\right)=i_{k}$ ).

(2) If $\pi_{1} a_{1} \pi_{2} a_{2} \cdots \pi_{n} a_{n} \pi_{n+1} \neq 0$, then

$$
\pi_{1} a_{1} \pi_{2} a_{2} \cdots \pi_{n} a_{n} \pi_{n+1}=\left\langle\pi_{1}^{-1}\left(i_{1}\right), \pi_{n+1}\left(\lambda_{n}\right)\right\rangle .
$$

Now, we prove Theorem 5

Proof of Theorem 5. For every simple graph we exhibit two terms over $M_{2}\left(Z_{2}\right)$, whose lengths are polynomial in the size of the graph, such that the graph is not 6 -colorable if and only if the two terms are equivalent. Thus, we reduce the graph 6-coloring problem into TERM-EQ $M_{2}\left(Z_{2}\right)$. Let $\boldsymbol{\Gamma}=\boldsymbol{\Gamma}(V, E)$ be a simple graph (with no loops and double edges). Notice that any possible isolated vertex can be ignored, so we can assume that the graph does not contain isolated vertices. For every vertex $j \in V$ we introduce a vertex-variable $v_{j}$ and for every edge $i \in E$ we introduce an edge-variable $e_{i}$. We define a few terms. Let

$$
\begin{aligned}
P & =\prod_{i \in E}\left(x w_{i}^{4}\right)^{6}, \\
Q & =\prod_{i \in E}\left(x w_{i}^{3}\right)^{6}, \\
H & =\prod_{(i, j) \in E^{2}}\left(w_{i} w_{j} w_{i} w_{j}^{2} w_{i}^{2}\right)^{6} .
\end{aligned}
$$

Here

$$
w_{i}=e_{i}^{5} v_{j} v_{k}^{5} e_{i} v_{k} v_{j}^{5}
$$

where $i$ is the edge connecting the vertices $j$ and $k$. The arguments will neither depend on the order of $v_{j}$ and $v_{k}$ in the definition of $w_{i}$ nor on the order in which the product defining $H$ is arranged. The products $P$ and $Q$ are running through the edges of $\boldsymbol{\Gamma}$ in the same order. Finally, let

$$
\begin{aligned}
p & =\operatorname{PPPxH}, \\
q & =\operatorname{PQPxH} .
\end{aligned}
$$

We claim that $p \equiv q$ if and only if $\boldsymbol{\Gamma}$ is not 6 -colorable. 
For this, first we analyze the expression $w_{i}=e_{i}^{5} v_{j} v_{k}^{5} e_{i} v_{k} v_{j}^{5}$. If the variables $e_{i}, v_{j}, v_{k}$ are all from the group $S_{3}$, then $w_{i}=e_{i}^{-1} v_{j} v_{k}^{-1} e_{i} v_{k} v_{j}^{-1}=\left[e_{i}, v_{k} v_{j}^{-1}\right]$, that is, the commutator of the group elements $e_{i}$ and $v_{k} v_{j}^{-1}$. In $S_{3}$ the commutator subgroup is $A_{3}=\{i d,(1,2,3),(1,3,2)\}$. Since the centre of $S_{3}$ is trivial, we have the following.

Lemma 10. Fix $i=\{j, k\} \in E$. Let us assume $v_{j}, v_{k}, e_{i}, a \in S_{3}$ and put $w_{i}=$ $\left[e_{i}, v_{k} v_{j}^{-1}\right]$. Then

(1) $a^{6}=i d$;

(2) $w_{i} \in A_{3}$, the commutator subgroup of $S_{3}$;

(3) $w_{i}^{3}=i d$;

(4) $w_{i}^{4}=w_{i}$;

(5) $w_{i}$ stabilizes 1 if and only if $w_{i}=i d$.

(6) For $u, v \in S_{3}$ there is an $e \in S_{3}$ such that $w=\left[e, u v^{-1}\right] \neq i d$, if and only if $u v^{-1} \neq i d$, that is, if and only if $u \neq v$.

(7) If $w_{i} \in S_{3} \backslash\{i d\}$, then the set $\left\{w_{i}, w_{i}^{2}, i d\right\}$ is transitive on $\{1,2,3\}$.

(8) If $w_{i} \in S_{3} \backslash\{i d\}$, and $s \in S_{A}$, then $s w_{i} s w_{i}^{2} s^{2}=0$.

Note that there exists at least one edge or vertex variable taking a value from $S_{A}$ if and only if there exists at least one word $w_{i}$ such that the value of $w_{i}$ is in $S_{A}$.

We will distinguish some cases that are described in Table1.

TABLE 1. The four different cases

\begin{tabular}{|c|c|c|c|c|}
\hline & \multirow{3}{*}{$\begin{array}{l}x \in S_{3} \\
\end{array}$} & \multirow{2}{*}{\multicolumn{2}{|c|}{$\overline{\langle i, \lambda\rangle=x \in S_{A}}$}} \\
\hline & & & & \\
\hline & & & $i \neq \lambda$ & $i=\lambda$ \\
\hline \multirow{2}{*}{$\exists w_{j} \in S_{A}$} & $\exists w_{i} \in S_{3} \backslash\{i d\}$ & \multicolumn{3}{|c|}{$\begin{array}{c}\text { Case } 2 \\
p=q=0\end{array}$} \\
\hline & $w_{i} \notin S_{A} \Rightarrow w_{i}=i d$ & \multicolumn{3}{|c|}{$\begin{array}{c}\text { Case } 3 \\
P=Q \text { hence } p=q\end{array}$} \\
\hline \multicolumn{2}{|r|}{$\forall w_{j} \in S_{3}$} & $\begin{array}{c}\text { Case } 1 \\
p=q=x\end{array}$ & $\begin{array}{c}\text { Case 4a } \\
q=P^{2} x= \\
P^{3} x=p\end{array}$ & $\begin{array}{l}\text { Case } 4 \mathrm{~b} \\
6 \text {-coloring }\end{array}$ \\
\hline
\end{tabular}

In the following we will show that except for Case $4 \mathrm{~b} p$ is always equivalent to $q$. Case 1: When all variables are in $S_{3}$. If all variables are from $S_{3}$, then $\left(x w_{i}^{k}\right)^{6}=$ $i d$ for every edge and $\left(w_{i} w_{j} w_{i} w_{j}^{2} w_{i}^{2}\right)^{6}=i d$ for every pair of edges, hence both terms are equal to $x$.

Case 2: When there exists $w_{j} \in S_{A}$ and there is an $i$ such that $w_{i} \in S_{3} \backslash\{i d\}$. The last item of Lemma 10 says the following: If there is an edge $j$, such that $w_{j} \in S_{3} \backslash\{i d\}$ and at least one $w_{j} \in S_{A}$, then $H=0$, and so $p=q=0$.

Case 3: When there exists $w_{j} \in S_{A}$ and besides $w_{i} \in S_{A} \cup\{i d\}$ for every $i \in E$. In both cases $w_{i}^{4}=w_{i}^{3}=w_{i}^{2}$, hence the two terms are equal.

Case 4: When $w_{i} \in S_{3}$ for every $i \in E$ and $x \in S_{A}$.

a) First, let $x=\langle i, \lambda\rangle$, where $i \neq \lambda$. In this case by item 3 of Lemma 10 $H^{3}=i d$ and so either $P=0$ or both sides are equal to $x$, hence the equation is obvious. 
b) Finally, without loss of generality, we may assume that $x=\langle 1,1\rangle$. Now, $p=$ $P P P x$ and $q=P Q P x$ and - because of item 3 of Lemma 10- $Q=\langle 1,1\rangle^{k}=0$, where $k \geq 2$, hence $q=0$. Thus $p \neq q$ if and only if there is a substitution, where $p=P P P x \neq 0$, by item 4 of Lemma 10 that holds if and only if $x w_{1} x w_{2} x \cdots w_{k} x \neq 0$. By Lemmas 8 and 9 we get that it is true if and only if none of the $w_{i}$-s stabilize 1 , which is by item 5 of Lemma 10 equivalent to $w_{i} \neq i d$. Recall that $w_{i}=e_{i}^{-1} v_{j} v_{k}^{-1} e_{i} v_{k} v_{j}^{-1}=\left[e_{i}, v_{k} v_{j}^{-1}\right]$, where $e_{i}$ is the edge variable and $v_{k}$ and $v_{j}$ are the elements assigned to the endpoints of $e_{i}$. According to item 6 of Lemma 10 we can choose an $e_{i} \in S_{3}$ such that $w_{i} \neq i d$ if and only if $v_{k} \neq v_{j}$, that is, if and only if the group elements assigned to the neighbor vertices are distinct, that is, if and only if $\boldsymbol{\Gamma}$ is 6 -colorable.

Corollary 11. TERM-EQ and $\mathrm{TERM}_{\Sigma^{-}} \mathrm{EQ}$ are coNP-complete for the ring $M_{2}\left(Z_{2}\right)$.

Theorem 12. There exists a 13 element semigroup $T$ for which the TERM-EQ problem is coNP-complete.

Proof. Namely, let $T=A_{3} \cup S_{A}$ be a subsemigroup of $M_{2}\left(Z_{2}\right)$. The proof is based on the same idea as the case of $M_{2}\left(Z_{2}\right)$ : for an arbitrary simple graph $\boldsymbol{\Gamma}$ with no isolated vertices we define the same polynomials as we did in the case of $M_{2}\left(Z_{2}\right)$ with the difference that here we let $w_{i}=v_{j} v_{k}^{-1}$. We will prove that $p \not \equiv q$ if and only if $\Gamma$ is 3 -colorable. We can claim a similar statement to Lemma 10.

Lemma 13. Let us assume that $w_{i} \in A_{3}$.

(1) $w_{i}^{3}=i d$;

(2) $w_{i}^{4}=w_{i}$;

(3) $w_{i}$ stabilizes 1 if and only if $w_{i}=i d$, i.e. $v_{j} \neq v_{k}$.

(4) If $w_{i} \in A_{3} \backslash\{i d\}$, then the set $\left\{w_{i}, w_{i}^{2}, i d\right\}$ is transitive on $\{1,2,3\}$.

(5) If $w_{i} \in A_{3} \backslash\{i d\}$, and $s \in S_{A}$, then $s w_{i} s w_{i}^{2} s^{2}=0$.

Accordingly, we can distinguish the same cases and in these cases except for Case $4 \mathrm{~b}$ the proof is word-by-word the same as for $M_{2}\left(Z_{2}\right)$. For Case $4 \mathrm{~b}$ again we may assume without loss of generality that $x=\langle 1,1\rangle$. Here by item 1 of Lemma 13 . $q=0 . p=P P P x \neq q=0$ holds if and only if $x w_{1} x w_{2} x \cdots w_{k} x \neq 0$. This is true if and only if none of the $w_{i}$-s stabilize 1 , which is by item 3 of Lemma 13 equivalent to $v_{j} \neq v_{k}$, that is, if and only if the group elements assigned to the neighboring vertices are distinct, that is, if and only if $\boldsymbol{\Gamma}$ is 3-colorable.

\section{FURTHER REMARKS}

At this point the following two problems arise.

Problem 1. Find the smallest semigroup for which the TERM-EQ is coNPcomplete.

Problem 2. Find the computational complexity of TERM-EQ for the semigroup $M_{n}(F)$.

However, it is not clear from the final version of the paper. During the proof we had the following interesting problem, which is the generalization of $\mathrm{EQN}^{*}$ in some sense. 
Problem 3. Given two sets of words $\left\{w_{1}, w_{2}, \ldots, w_{n}\right\}$ and $\left\{v_{1}, v_{2}, \ldots, v_{m}\right\}$ over $G \leq S_{k}$, the symmetric group acting on the set $\Omega=\{1,2, \ldots, k\}$. For an evaluation of the variables and for $l \in \Omega$ let $I_{l}=\left\{w_{1}(l), w_{2}(l), \ldots, w_{n}(l)\right\}$ and $J_{l}=$ $\left\{v_{1}(l), v_{2}(l), \ldots, v_{m}(l)\right\}$. Find the complexity of the question whether the setequation system $I_{1}=J_{1}, I_{2}=J_{2}, \ldots, I_{k}=J_{k}$ holds for every evaluation.

If $n=m=1$, then we get the word-problem for the fixed permutation group.

\section{ACKNOWLEDGEMENTS}

The research of the authors was supported by the Hungarian National Fundation for Scientific Research, Grant F32325, T043671 and T038059.

\section{REFERENCES}

1. D. M. Barrington, P. McKenzie, C. Moore, P. Tesson, and D. Thérien, Equation satisfiability and program satisfiability for finite monoids, Math. Found. Comp. Sci. (2000, Bratislava), 127-181. MR 1844742 (2002f:68053)

2. S. Burris and J. Lawrence, The equivalence problem for finite rings, Journal of Symbolic Computation 15 (1993), 67-71. MR 1210448 (94c:16030)

3. H. Hunt and R. Stearns, The complexity for equivalence for commutative rings, Journal of Symbolic Computation 10 (1990), 411-436. MR 1087713 (92g:68062)

4. A. Kisieliewicz, personal communication, 2002.

5. J. Lawrence and R. Willard, The complexity of solving polynomial equations over finite rings, manuscript, 1997.

6. V. Yu. Popov and M. V. Volkov, Complexity of checking identities and quasi-identities in finite semigroups, Journal of Symblic logic (to appear).

7. S. Seif and Cs. Szabó, The computational complexity of checking identites in simple semigroups and matrix semigroups over finite fields, Semigroup Forum (to appear 2002).

Department of Algebra and Number Theory, Eötvös Loránd University, 1117 BuDAPEST, PÁzmány PÉter SÉtány 1/C, Hungary

E-mail address: csaba@cs.elte.hu

Department of Algebra and Number Theory, Eötvös Loránd University, 1117 BuDAPEST, PÁZMÁNy PÉTer SÉtÁNy 1/C, Hungary

E-mail address: wera13@cs.elte.hu 